\title{
Carnets
}

Revue électronique d'études françaises de l'APEF

Deuxième série - 19 | 2020

Petite fabrique d'interprètes

\section{Interpréter la tentation du spirituel dans quelques romans contemporains français}

\section{Catherine Grall}

\section{OpenEdition}

1 Journals

\section{Édition électronique}

URL : http://journals.openedition.org/carnets/11687

DOI : 10.4000/carnets. 11687

ISSN : 1646-7698

Éditeur

APEF

\section{Référence électronique}

Catherine Grall, «Interpréter la tentation du spirituel dans quelques romans contemporains français », Carnets [En ligne], Deuxième série - 19 | 2020, mis en ligne le 31 mai 2020, consulté le 23 décembre 2020. URL : http://journals.openedition.org/carnets/11687; DOI : https://doi.org/10.4000/carnets. 11687

Ce document a été généré automatiquement le 23 décembre 2020.

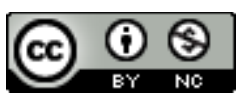

Carnets est mis à disposition selon les termes de la licence Creative Commons - Atribution - Pas d'utilisation commerciale 4.0 International. 


\title{
Interpréter la tentation du spirituel dans quelques romans contemporains français
}

\author{
Catherine Grall
}

1 Le besoin de spirituel paraît s'affirmer de plus en plus dans le monde occidental d'aujourd'hui : pape populaire rencontré par de nombreux chefs d'État ${ }^{1}$, crises de l'Islam, pays anciennement communistes revenant à la religion ${ }^{2}$, innombrables méthodes de développement personnel mettant l'accent sur l'importance de se détacher de la société matérialiste et technophile stressante, immense succès des pratiques méditatives, vogue du chamanisme dans les arts, retour de la tendance New Age... Depuis la fin du XXe siècle, la littérature de fiction, après de multiples « ères du soupçon» (Sarraute, 1956), et sous le coup de plusieurs événements historiques, a opéré un retour vers le réalisme, avec des dimensions volontiers testimoniales et documentaires - quelles formes prend alors ce « retour du spirituel », qui n'équivaut pas cependant au «réenchantement du monde » que les critiques de Weber (1921) 3 notent parfois ? Sans prétendre à un tableau exhaustif de la littérature contemporaine de langue française, nous examinerons la tendance de quelques œuvres à exprimer un désir de transcendance sans prosélytisme, ou à emprunter au moins les formes de la spiritualité, tout en représentant le monde contemporain, et en tenant un discours sur celui-ci. Les situations réalistes contemporaines semblent y résulter d'une sécularisation où l'humain ne s'épanouit plus, manque de valeurs éthiques, voire d'identité, et d'inscription dans des communautés - bref, les mondes dépeints par ces auteurs relèvent de ce qu'Habermas (2008:5) a pu appeler le "post séculier ", mais un post séculier en suspens, où les aspirations religieuses n'entrent pas en synergie avec le séculier. 


\section{Variations sur le religieux : situation de la littérature du XXIe siècle}

2 L'inscription du spirituel ou du religieux dans la littérature peut prendre des formes privilégiées. Selon Thomas Pavel, les romans qui « construisent un vaste univers fictif fort différent du monde de la vie quotidienne", utilisent une méthode «idéographique», selon laquelle "l'univers imaginaire est façonné par une idée unificatrice que la multitude d'épisodes évoquent inlassablement (... idée unificatrice susceptible,) dans son abstraction et son étrangeté, d'éclairer de manière globale l'univers tenu pour réel. » (Pavel, 2003 : 111). Pareilles écritures orientent volontiers le lecteur vers un sens spirituel, éventuellement religieux, qui se complique à partir du XVIIIe siècle, lorsque les romanciers inscrivent les valeurs transcendantes dans des mondes désormais vraisemblables, ie susceptibles de renvoyer directement à l'expérience quotidienne. Si le XXe siècle a continué, après les crises romantiques, symbolistes et avant-gardistes, d'accumuler les crises du roman, en particulier à l'encontre de ses ambitions réalistes, il est aussi, surtout dans sa seconde moitié, le siècle qui a vu revenir, sans naïveté mimétique, le réalisme. Écritures du témoignage, en particulier suite aux traumatismes collectifs, écriture du reportage (du gonzo journalism à la factographie ${ }^{4}$, en passant par le récit de voyage), formes autobiographiques (démocratisation de l'écriture, y compris, ces derniers temps, sur le web) n'en présentent pas moins volontiers une dimension morale. Narrer sa vie est susceptible de former la personne qui écrit, d'aider le lecteur à se situer par rapport à la parole littéraire, voire à prendre la plume (on développe les ateliers d'écriture en ce sens ${ }^{5}$ ) ; c'est aussi passer la réalité contemporaine au filtre de perceptions et de voix singulières, et rendre ainsi accessible ce qu'on ne vit pas soi-même. L'écriture testimoniale produit aussi cet effet, en y ajoutant la prise de conscience des pans les plus noirs de l'histoire (génocide, colonialisme...) ou des injustices sociales (ségrégation, homophobie...). L'écriture de reportage, enfin, ou à vertu documentaire, dans un dernier sursaut de méfiance par rapport à la fiction, pousse celle-ci dans ses limites et informe le lecteur, d'une manière plus sensible, voire plus "authentique », que les essais ou la presse. L'éthique du lecteur étant concernée - plus sur un plan privé que sous forme idéologique (religieuse ou politique) et l'idéographie semble donc percer à nouveau, du sein même de l'écriture empirique.

Ces dimensions morales ont pénétré la littérature et la critique littéraire de langue française après les littératures et théories anglo-saxonnes: les Cultural studies, les Gender studies, les Queer studies, les Postcolonial studies, voire les Animal studies, les théories du care sont encore peu représentées à l'université française, alors que de très nombreux discours sociaux et politiques y ressortissent. Durée persistante d'approches textualistes des œuvres, alors que le «Nouveau roman" était le dernier courant français à avoir influencé les littératures étrangères ? Les récents adeptes des X-studies se sont réclamés de plusieurs philosophes obsédés par le texte, mais Derrida, Foucault, Deleuze ressaisissaient aussi les questions sociales... Le souci occidental pour l'écologie planétaire est aujourd'hui représenté depuis la France par un penseur comme Bruno Latour, et les philosophes de la "vie bonne » se multiplient (dans le sillage de Pierre Hadot relisant l'antiquité ${ }^{6}$, ou de la philosophie analytique et de la philosophie de l'esprit) si bien que, à plusieurs reprises, on a redemandé «à quoi sert la littérature ? »7 
4 Pareilles préoccupations éthiques troublent de nombreux personnages contemporains de la littérature occidentale, au point de les «spectraliser. » La critique anglophone a en effet osé le terme de Spectral turn ${ }^{8}$ pour qualifier le caractère fantomatique de personnages revenant d'un ailleurs extraordinaire, de manière métaphorique ou pas, ou en dérive dans un univers qu'ils ne comprennent plus et ne parviennent plus à habiter. Ce sont, entre autres, les victimes de crimes passés qui hantent les mémoires et empêchent d'envisager un avenir - les personnages «hantés " peinant eux-mêmes à vivre dans ces conditions. Ces « spectres » d'un nouveau genre réclament souvent, plus ou moins directement, la justice. Or les religions sont par excellence des systèmes produisant des valeurs et faisant communauté. C'est dans cette mesure que le post séculier connote ces écritures et invitent à les analyser comme des symptômes sociaux et comme des perceptions contemporaines du monde - où les idéologies n'enthousiasment plus facilement, mais où le bon et le bien mobilisent encore.

\section{Manières de post séculier littéraire français}

5 Les questionnements métaphysiques, sur fond de troubles socio-économiques, de fin du «camp " communiste, de développement d'un néo-libéralisme sans pensée de l'humain, de malaises que les neurosciences ne suffisent pas à nommer, d'attentats terroristes, de multiculturalisme qui effraie produisent des personnages inquiets, souvent tentés par des mouvements spirituels. Il peut s'agir de la foi, ou de l'expérience souvent bouleversante de transcendances mal définies. Emmanuel Carrère (2010), après D'autres vies que la mienne, a publié récemment Le Royaume (Carrère, 2014), où il exposait ses années de foi et de pratique chrétiennes intenses; il était par ailleurs interviewé récemment sur France Culture ${ }^{9}$ pour expliquer sa pratique du yoga, dans la perspective d'un équilibre pas seulement corporel. Cette juxtaposition ne vise pas à choquer : il s'agit là d'un écrivain reporter, romancier et auteur d'autofiction, régulièrement en proie à des troubles névrotiques, en recherche de "vie bonne", et qui ne peut se contenter du mondain, dans un présent rationnalisé qui l'étouffe.

6 Cela se manifeste de façon plus discrète chez Jean Rolin qui, après une jeunesse maoïste (dans la Gauche Prolétarienne, qui, telle une Église, interdisait plaisirs sexuels, alcool et toutes autres drogues), est devenu reporter, écrivain aujourd'hui très prolixe, bourlingueur et jouisseur mélancolique. Toujours intéressé par la politique internationale, son personnage rapporte des enquêtes qui donnent à méditer sur les modes de vie les plus variés, en accordant une grande part au contexte, et en produisant une impression de vanité, relatives aux combats incessants des hommes. Auteur d'une enquête sur les chrétiens dans les territoires occupés (Rolin, 2003), de Savannah (Rolin, 2015), tombeau à une amie disparue, et d'Événements (Rolin, 2015), où des groupes armés d'obédience soi-disant religieuse mettent la France à feu et à sang, l'auteur se met en scène dans Joséphine, dont les dernières pages évoquent ses souvenirs de Dinard, marqués par la mort d'un oncle, la mort contemporaine d'une fillette, et les travaux un peu hamletiens de jardiniers pris pour des fossoyeurs, qui bêchent auprès d'un oratoire; Jean Rolin cite ainsi la prière inscrite sur une plaque funéraire, prière qui demande à la Vierge sa pitié pour les malheureux de ce monde ${ }^{10}$. Son personnage, dans Savannah comme dans Joséphine, choqué par la disparition de ses amies, et par les violences politiques et sociales qu'ils enregistraient tous les deux, semble avoir besoin d'une pause spirituelle dans son désespoir. À la fin de Zones (Rolin, 
1995 : 188-189) il comparait la piété «ostentatoire » d'une manifestation de chrétiens, qui lui rappelle les temps de l'Inquisition, à la prière intime, mieux tolérée, semblant ainsi revenir à la distinction entre sphère privée et sphère publique, c'est-à-dire à séparer la foi du politique.

7 Philippe Vasset, spécialiste en économie (il est le rédacteur en chef de la revue en ligne Intelligence), se passionne pour la constitution des légendes par le Vatican, et figure un narrateur en crise de foi dans le roman du même titre (Vasset, 2016), cependant que le personnage principal de La Conjuration (Vasset, 2013), écœuré par le monde du travail, fonde une secte sans but, mais avec rituels, qui grossit et hante les nuits urbaines. Yannick Haenel (2007), dans Cercles, figurait également un personnage en marge du monde du travail et en quête d'une " existence absolue ", tout comme le narrateur des Renards pâles (Haenel, 2013), qui finit par intégrer une secte de sans-papiers aux rituels africains.

8 Ces écrivains qui évoquent la foi ou figurent des simulacres d'expérience religieuse présentent des personnages fatigués de la vie mondaine et en quête de mondes meilleurs, ce qui n'est pas le cas des personnages de Houellebecq, qui assument plus ou moins facilement la condition séculière contemporaine dans ses dimensions les plus matérialistes, injustes et avides. Ce qui ne va pas sans nostalgie spiritualiste et peur du religieux "autre »: sentiment de ne pas savoir aimer, de ne pas être aimé, rêve de dépasser la mort (Les particules élémentaires), inquiétude que la collaboration d'institutions françaises imaginaires avec l'Islam radical enfonce comme jamais la société dans le machisme et la corruption (Houellebecq, 1998, 2015). Les romans pessimistes de Houellebecq n'envisagent pas le post séculier au sens constructif, ils expriment des retours au pouvoir religieux, au pire sens d'un Islam rigoriste et hypocrite.

9 Citons encore trois œuvres contemporaines écrites par des auteurs d'origine maghrébine, traitant elles aussi de l'Islam dans ses rapports à la vie quotidienne et à la société : Abdellatif Taïa, Kamel Daoud et Boualem Sansal, selon des écritures très différentes, critiquent en effet les aspects traditionnalistes et obscurantistes de la religion. Dans son roman Infidèles, Taïa dit d'abord la misère intellectuelle et matérielle de deux femmes marocaines, prostituées, maltraitées dans une société hypocrite et par un pouvoir politique très violent. Sa mère donne beaucoup d'amour au personnage principal, Jallal, avant de mourir en inspirée mystique, proposant ainsi une autre expérience de la religion dans le monde. Jallal découvrira l'Islam fondamentaliste en Belgique, auprès d'un jeune converti dont il tombe amoureux et qu'il suit, confondant geste d'amour romantique (suicide à deux) et attentat terroriste (allumage d'une ceinture d'explosifs à Casablanca, après une nuit passée dans la Grande Mosquée). Le dernier chapitre, intitulé « Dieu », fait parler celui-ci, assimilé à une Maryline Monroe transgenre, ce qui finit par renvoyer toute forme orthodoxe à un miroir aux alouettes.

Kamel Daoud (2016), animé par l'esprit curieux mais critique d'Edward Saïd à l'égard de Camus, répond à L'Étranger de celui-ci en dénonçant la cécité du colon et du narrateur, quant à la vie des Algériens (laissés sans nom par l'auteur français). Mais Daoud contrebalance cet aveuglement " impérialiste " à sa manière en dénonçant violemment le dogme religieux (en particulier dans le chapitre VII), à côté de l'inconscience et les malhonnêtetés politiques, économiques, urbanistiques, voire écologiques du gouvernement. Le romancier journaliste s'intéresse aussi au sort réservé aux femmes dans le milieu où évolue son narrateur qui accuse l'obscurantisme archaïque dont a été 
victime et vectrice sa mère, mais aussi les insultes, les fantasmes puritains contre les femmes en général dans le milieu " arabe ", comme les menaces incestueuses que fait peser sur elle le père de Meriem. Spiritualité et libéralisme dominateur sont ici renvoyés dos à dos, et l'absurde existentialiste camusien est devenu politique - seul un personnage féminin (Meriem, une Marie qui n'a rien d'une sainte vierge) paraît faire signe vers le futur.

11 La dystopie 2084 de Boualem Sansal (2015) met en scène une religion apparentée à l'Islam, désignée ouvertement comme la complice criminelle d'une dictature - mais la culture représente le dernier espoir (le personnage principal, Ati, enquête sur un peuple de renégats et apprend à remettre en cause sa société autoritaire).

12 À côté de ces fois chrétiennes et musulmanes, de ces embryons de sectes, Patrick Chamoiseau (2012) place plutôt son personnage dans une communion spirituelle avec la nature, dans L'Empreinte à Crusoe. Le complice négrier de Robinson est noir et une maladie l'a rendu furieux, au point que ses camarades l'ont abandonné sur une île déserte. Là, amnésique, il pense s'appeler Robinson, au vu des quelques affaires dont il dispose, et grâce auxquelles il colonise l'île, où il craint la présence d'un autre, avant de le désirer ardemment, puis de découvrir qu'il ne traque que lui-même. Pénétré de sagesse pythagoricienne (grâce à un livre issu du pseudo naufrage, qui remplace la Bible du roman de Daniel Defoe), il entreprend de mieux observer la nature, il libère les animaux domestiqués, se nourrit de cueillette sauvage et délaisse ses champs avant de mourir, retrouvé par son ancien camarade pris de remords, mais trop violemment indigné par les esclaves qu'il découvre dans la cale du bateau. L'écriture poétique de Chamoiseau rappelle le réalisme magique pour laisser s'exprimer une voix inspirée, qui réinvente le monde, l'amour, et le désir d'égalité respectueuse. Le spirituel est ici une forme de paganisme qui se donne pour dépassant les «cultures» qui divisent les hommes, mais il ne mène à aucune sociabilité.

Les références littéraires françaises convoquées correspondent assez bien aux divers usages de la notion de post séculier, telle qu'abordée dans la synthèse de Luca Mavelli et Fabio Petito (2012): le post séculier peut en effet renvoyer à des formes de résurgences du religieux, et c'est le cas de tous nos auteurs, et à la façon dont le politique peut inclure les éléments religieux. Ce second cas, non exclusif du premier, est en partie représenté par Houellebecq et Sansal sur le mode de la dystopie plus ou moins lointaine, beaucoup moins par Carrère, Rolin, ou Vasset (les éléments religieux de leurs romans ne présentant pas de dimension réellement politique, mais plutôt personnelle, méditative et sociocritique). Chez Taïa et Daoud, les politiques passées sont dénoncées (impérialisme et misère morale éventuellement antérieure), tout comme une compréhension obscurantiste de l'Islam, en particulier à l'égard des femmes. Les romans de ces deux auteurs relèvent donc aussi en partie de l'acception plus critique du post séculier que relèvent enfin les deux chercheurs italiens : celui-ci correspondrait en effet encore à la mise en cause des pouvoirs séculiers, incapables d'assurer réellement les notions de démocratie, d'égalité, d'inclusion et de justice. Soumission de Houellebecq va sans doute jusque-là (il dénonce la faiblesse d'une démocratie qui renonce à l'égalité), mais cette critique apparait aussi dans Les Renards pâles de Haenel (prise en compte de la marginalisation des immigrés du continent africain, susceptibles d'apporter un renouveau spirituel et politique).

La dimension spirituelle, dans tous ces cas, apparaît comme la cause ou le symptôme de la difficulté à exister dans l'ici et maintenant, avec ses semblables. 


\section{Aspiration à (re)faire communauté}

\section{d} les villes (Vasset, Haenel), dans la géopolitique internationale (Rolin, Daoud), dans des politiques nationales faibles et hypocrites, ou dictatoriales (Houellebecq, Sansal, Riboulet, Daoud), sur une île déserte où leur identité même fait problème (Chamoiseau), ils sont condamnés à rester seuls, sans passé toujours assumable et sans idéologie prometteuse de futur. Les romans finissent d'ailleurs sur le renoncement à marier le transcendant et le mondain, pour un post séculier progressiste - au mieux, ils présentent des fins ouvertes. Leurs personnages rêvent de formes communautaires peu viables, que les romans évoquent parfois seulement dans les dernières pages, sans leur donner d'aboutissement. Les Renards pâles finit par une manifestation aux accents religieux africains sur la Place de la République...sans qu'on en connaisse l'issue. Le narrateur de Jean Rolin ne paraît pas espérer l'intégration par les politiques des tolérances et de l'amour prônés par les religions. La secte originale imaginée par Philippe Vasset dans La Conjuration grossit sans pouvoir mener à rien jusque dans les dernières pages, qui s'apparentent à une apocalypse douce: le chapitre 14 («Les conjurés sont dans la ville comme dans un cloître : leur circulation est prière ») achève la partie II, qui a elle-même pour titre une citation de la Règle de Saint-Benoît : "Le silence devra être absolu, et on n'entendra aucun chuchotement ni aucune voix, si ce n'est celle du lecteur ». Le narrateur y conte poétiquement, mais non sans ironie, comme la citation mettant le lecteur profane aussi en exergue, la victoire des adeptes dans l'espace urbain : leur parole sera chargée de tout dans la cité, si bien qu' « ils feront de la ville un infini murmurant. Et la langue elle-même deviendra paysage. ${ }^{11}$ » L'ouverture écologique, philosophique et poétique que vit le héros malheureux de Chamoiseau, après sa phase d'exploitant de l'île, se referme brutalement sur son assassinat. Si une expérience religieuse du monde semble bien s'emparer de lui, lui permettre de retrouver une manière de paradis terrestre sans désir de domination, la première tentative pour renouer avec ses semblables dit l'inhumanité immorale de ceux-ci (procès postcolonial connu de Robinson Crusoe). Pas de vie possible avec les autres hommes.

On observe une orientation semblable dans un petit roman italien récent, qui fait d'une aspiration spirituelle un motif de changement de vie, que l'auteur, hélas, ne développe pas, ratant ainsi une dimension morale très actuelle - ici les Women studies, très liées à la pensée de la communauté et de l'inclusion. Dans Noli me tangere, en effet, le personnage principal d'Andrea Camilleri (2018) enquête sur une femme mystérieuse, qui a fait des recherches sur Fra Angelico, particulièrement sur son illustration du 
"Noli me tangere", femme sujette à des sautes d'humeur, intelligente, croqueuse d'hommes qui ne trouve jamais satisfaction. Elle finit par s'engager dans une association humanitaire, et l'auteur achève son roman par une postface où il précise qu'il a voulu faire le portrait d'une amie, tuée par les malheureux Indiens qu'elle voulait aider. Cette esquisse d'hagiographie moderne ne laisse quasiment jamais la parole au personnage féminin et ne l'évoque comme agissante que dans un futur possible, tout en barrant celui-ci par la référence à une réalité qui semble conclure à l'échec cynique de la femme charitable. L'image de Marie-Madeleine, la supposée prostituée qui suivit le Christ jusqu'au bout et vit la première son retour d'entre les morts, ne sert pas de seule métaphore psychologique : la chercheuse est fascinée par le rapport qu'eut celle-ci avec le fils de Dieu, et semble orienter sa vie dans un sens proche, en pratiquant une conversion active dans la société. Mais l'auteur préfère romancer ses insatisfactions sexuelles que développer cette activité, qui pourrait donner lieu à un challenge politique (cf le débat actuel, en grande partie, sur la fonction des ONG humanitaires, sur leur positionnement par rapport aux différents régimes, et aux différentes religions, sur les actions qu'elles mènent et dont les États ne sont plus capables): que serait une société de la bienfaisance, fondée en expérience et en culture ? Celle-ci ne peut-elle que rester marginale ? Camilleri reste lui aussi en-deçà d'une troisième étape de la dialectique qui nuancerait l'opposition entre un monde séculier largement libéral et le religieux.

La religion a depuis toujours été associée au politique, dans ses manifestations mondaines, et les idéologies athées ont emprunté parfois ses formes. Nous avons signalé plus haut le "purisme", pour ne pas dire "puritanisme» de la Gauche Prolétarienne, dont les frères Rolin font une critique dans ces termes, et c'est sans surprise que l'on peut trouver dans des récits très connotés par l'histoire sociopolitique des personnages contemporains au statut fantomatique (et donc à dimension morale critique). Didier Castino (2015) fait ainsi parler le spectre d'un ouvrier tué par une machine. Son personnage a cru dans une solidarité syndicale qui créait une véritable famille sociale, faute d'adopter une religion, et il cherche à transmettre à son fils une mémoire ouvrière sacralisée par la mort, ainsi qu'une mémoire des luttes politiques, quand celui-ci paraît finalement avoir accepté le compromis de la société de consommation, communauté dont les valeurs sont opposées à celles du père, mais pas dialectisées avec celles-ci par le roman.

L'échec de mouvements de la gauche radicale a laissé plus d'un écrivain sans horizon, et l'idéal politique tend à laisser la place à un autre idéal : si l'écologie n'est pas assez puissante, si la nation ne forge pas à leurs yeux de communauté imaginaire suffisante (Anderson, 1996), il reste le repli sur soi, avec de vagues espoirs irrationnels, nourris de nostalgie $^{12}$, voire le refuge dans des politiques religieuses radicales, comme l'activisme terroriste ${ }^{13}$. Mathieu Riboulet (2015), dans Entre les deux il n'y a rien ${ }^{14}$, exprime son regret d'être né trop tard pour une vraie révolution et explique avoir fait le choix d'une homosexualité tapageuse et active, comme pour suppléer à ce manque de réalisation sociale - dans une sorte de roman autobiographique scandé par le rappel des amis morts. La RAF (Rote Armee Fraktion) qu'il met en scène, et que cite son titre, également travaillée par Alban Lefranc (2006), tuait pour une vie meilleure et contre une société gangrenée par l'esprit de consommation sans morale.

20 Comment comprendre l'expression nostalgique de ces aspirations spirituelles, exprimée parfois avec une distance prudente? Il semble que ces œuvres, derrière 
l'aspiration déçue à des communautés éthiques, partagent une critique du monde où l'individu est brimé dans ses désirs d'actions positives et jugées morales. Concrètement, ces auteurs remettent en cause des formes variées d'antilibéralisme.

\section{Post séculier, communauté et libéralisme}

21 Les œuvres du corpus mettent en effet en scène un monde occidental contemporain où l'individu se trouve perdu; le passé a pu sembler révolutionnaire, c'est-à-dire propice aux croyances en des changements radicaux de sociétés jugées trop matérialistes (bouleversements religieux et politiques), mais les utopies ont été déçues. L'esprit libéral qui l'a emporté dans les sociétés occidentales, et qui se pose en objet de désir pour beaucoup d'autres, ne nourrit plus guère d'espoir pour l'individu et peine à faire communauté, en particulier depuis que l'ouverture au monde a fait éclater les injustices du colonialisme et que les sociétés occidentales ne proposent plus à leurs membres de modèles de "vie bonnes ». La philosophie libérale a d'abord favorisé l'épanouissement de l'individu, compris comme membre d'une société, et cette pensée a été associée au progrès et à la sécularisation (confiance en l'individu autonome et responsable, loin de tout Léviathan). Catherine Audard (2009: 727) rappelle ainsi que : "le libéralisme a été avant tout une réflexion sur les conditions de la paix civile et une réponse à la question : "comment vivre ensemble? ", depuis Locke et Hume jusqu'à John Stuart Mill et à T. H. Green, de John Dewey à John Rawls", interrogation qui trouvait une réponse non pas tant dans la foi placée en la raison, mais dans une pensée de l'intérêt commun, qui résulterait de l'ensemble des intérêts singuliers bien équilibrés. Dans son célèbre Rise of the Novel, Ian Watt, étudiant en particulier le roman de Daniel Defoe, postulait une concomitance entre, d'une part, la société anglaise toujours plus sécularisée, avec des lecteurs toujours plus nombreux et plus modestes, cherchant du divertissement, et, d'autre part, le puritanisme. L'étude de 1957 prolonge en effet les thèses weberienne sur l'Éthique protestante et l'esprit du capitalisme (Weber, 1964 [1920]). Le résultat littéraire en était la peinture d'un contexte socioéconomique contemporain, avec des personnages ordinaires, susceptibles d'ordonner leur vie, en vue d'une réussite en ce monde, réussite matérielle interprétable comme le signe d'une élection divine. Daniel Defoe, avec Robinson Crusoe comme avec Moll Flanders, plonge des personnages démunis dans un univers dont ils franchissent les obstacles très vraisemblables (construire un abri, trouver sa nourriture, pour un marin échoué sur une île déserte, survivre en tant que femme seule à Londres à la fin du XVIIe s.), non sans l'aide de Dieu (Robinson a sauvé une Bible du naufrage, et Moll se convertit en prison, avant de devenir à sa manière une exploitante, dans les colonies britanniques). La pensée économique et sociale semblait alors se marier parfaitement avec les exigences religieuses.

Le XXe siècle, en particulier la pensée marxiste, a progressivement dénoncé cette croyance de la réalisation de l'individu moderne par le travail et la consommation : les deux correspondent trop largement à des formes d'exploitation. La dimension socialisante du libéralisme a été toujours plus mise de côté par ses développements économiques et politiques, en particulier sous sa forme néo-libérale, alors même que les gouvernements occidentaux se dégageaient d'un marxisme dévoyé. Le roman réaliste du XIXe enregistrait déjà des figures d'arrivistes et les conséquences sociales et historiques de l'industrialisation et des concurrences internationales; le XXe siècle 
multipliait les crises (sociétales et artistiques). Le XXIe, avec ses rétrospections morales, pointées entre autres par les postcolonial studies, redit la nécessité de représenter le monde contemporain, en y incluant des crises des valeurs qui ne peuvent plus aboutir à des utopies politiques et se cherchent de nouveaux vecteurs : les auteurs " post séculiers » abordent l'éthique et le spirituel au sein d'un monde soucieux d'égalité de droit entre les hommes d'un même pays ou de plusieurs pays...avec des résultats essentiellement pessimistes. Le "cadre immanent», que Charles Taylor définit par un espace social animé par un temps séculier et régi par la rationalité instrumentale (Taylor, 2011: 920), qui a paradoxalement été induit par l'individualisation de la foi (en particulier depuis la Réforme) est devenu insupportable, et fonde désormais un individualisme en quête de valeurs, mais qui peine à produire du commun.

Dans les pays anglo-saxons, de tradition protestante au sens le plus large (évangélique), cette nostalgie peut prendre des formes plus positives, qu'illustrent de grands romans affrontant directement la question sociale, sous la forme contemporaine et globale du multiculturalisme. Le post séculier analysé par Manav Ratti (2013) dans The Postsecular Imagination. Postcolonialism, Religion and Literature correspond à la critique des politiques séculières en réalité incapables de soutenir des sociétés multiconfessionnelles et agnostiques ou athées (cf par excellence la laïcité à la française). Citant William Connolly, et retrouvant Salman Rushdie, le chercheur paraît espérer une religion sans dieu et une politique sans dogme, qui sauvegarderaient l'enthousiasme spirituel de chacun, sans s'opposer à l'érection d'une communauté hétérogène, ce qu'il retrouve dans l'analyse de quelques grands romans contemporains, souvent placés dans la World Literature $^{15}$. D'une certaine manière, l'aspect sociopolitique que le libéralisme a oublié de penser se trouve récupéré par une poétique globalisante, ie qui amène les personnages à traverser les frontières nationales et linguistiques, voire celles qui séparent fiction et non fiction, littéralisme et métaphore.

Or, le corpus francophone présenté ici se risque finalement assez peu à pareilles manœuvres: les Africains de Haenel ne disent pas beaucoup plus leur projet que la femme dans le roman de Camilleri. Les voyages érudits et ironiques de Rolin concluent souvent sur le personnage de l'enquêteur blasé, parfois triste, ou sur des personnages originaux, mais guère sur des liens larges. Vasset brosse des portraits féroce ou victimaires, pas combattants, et les rêves demeurent des phrases : jamais la dimension poétique ne relève d'un réalisme magique favorable aux changements de perspectives et de monde. C'est davantage le cas chez Patrick Chamoiseau, mais il ne partage pas les projets (peut-être utopiques ou trop « littéraires ») d'un Glissant sur le Tout-monde, et L'Empreinte à Crusoé s'achève sur un hommage érudit et baroque à un Defoe revitalisé par l'Antique, mais pas sur des promesses de vie commune. Les écrivains français maghrébins ne nous laissent guère plus d'espoir, entre la dystopie de Sansal, qui n'est que l'exact envers de celle de Houellebecq, la réécriture de Daoud qui reste, plus que Chamoiseau, un peu prisonnier de son hypotexte, et les Infidèles de Taïa, autant tourné vers la clôture que le beau texte de Riboulet.

\section{Quelle(s) poétiques pour le post séculier?}

Le multiculturalisme est-il en défaut dans notre corpus, dans la littérature française en général, et cela empêche-t-il une pensée littéraire de la société comme susceptible 
d'articuler le "cadre immanent» et les enthousiasmes éthiques religieux ? C'est possible. Nous voudrions surtout ajouter que pareille pensée des différences exige aussi des écritures peut-être moins prosaïques. Le « réalisme » retrouvé dès les années 1980 dans la littérature occidentale n'a pas évacué les doutes sur la perception du monde, sur la représentation et sur l'expression de celui-ci. La mise en scène de la narration, le portrait insistant d'un narrateur faible, d'un centre focalisateur instable, d'intrigues où l'aléatoire déconcerte, sont utilisés par nos auteurs, qui se départissent encore rarement d'une ironie moderne et postmoderne, ie déconstructrice et narcissique. Ces œuvres ressortissent certes à une forme de « spectralité » et quelques caractéristiques communes peuvent être dégagés en ce sens. Ainsi, les récits connaissent volontiers des évolutions temporelles et spatiales brouillées : les errances de Jean Rolin se retrouvent dans les errances urbaines des personnages de Haenel et de Vasset, ainsi que dans les parcours en trajectoires brisées de ceux de Chamoiseau, de Daoud ou de Taïa. Les boucles et les répétitions sont fréquentes, voire les pertes de repère. Les communications entre les personnages sont difficiles (misanthropie pathologique des personnages des Renards pâles, de La Conjuration, isolement du narrateur de Taïa, soupçon sur tous les autres personnages de la part de celui de Daoud, soliloques de celui de Chamoiseau, névroses houellebecquiennes...). L'intrigue, douloureuse, laisse volontiers la place à des passages méditatifs, à des retours critiques, à des questionnements - et l'on remarque alors une touche picaresque -, voire à des plaintes lyriques (Haenel, Chamoiseau, Taïa...) - où perce plutôt un accent néo-romantique (voir l'écriture du tombeau de Riboulet). Les descriptions constituent une autre manière de malmener un peu le fil narratif : l'espace prolonge le malaise ou le délire du personnage en manque de transcendance et de communauté (voir en particulier l'écriture poétique de Chamoiseau, mais aussi Riboulet et de Vasset:longues phrases, vocabulaire spirituel, suspension du temps, ...). Mais ils manquent encore les profondeurs du dialogisme et de l'espace, voire une poétique de l'image ou même la récupération contemporaine des intrigues romanesques que pratiquent par exemple les auteurs cités par Manav Ratti, ou une Toni Morrisson, voire, pour rester en Europe, d'un W. G. Sebald. L'écrivain allemand - symptôme d'un vieux continent dont le modèle ne convainc plus? -, riche pourvoyeur en personnages spectraux (il joue aussi de la photographie qui perturbe la perception du lecteur), excelle dans l'écriture d'un présent rendu invivable par les crimes passés, en travaillant des scènes de révélation qui font songer à des épiphanies, plus ou moins réussies. Thomas Richard Bell (2015), à propos de cet auteur, mais aussi à propos d'œuvres de Daniel Kehlmann et de Peter Handke, relève des traces de "transcendance post séculière", qu'il identifie à l'articulation qu'effectua Schleiermacher entre Lumières et conception intuitiviste de la religion. Or, ces analyses peinent à s'appliquer à notre corpus. L'émotion s'y trouve, dans des envolées lyriques chez Vasset (mais ironiques), toujours narcissiques chez Carrère, assombries par un ton macabre (chez Riboulet ou chez Rolin), voire par un onirisme qui reste incomplet (fin du roman de Haenel) ou un humour incongru (fin du roman de Taïa).

Il ne s'agit pas tant ici de porter des jugements de valeur sur les écritures de ces romanciers français que de revenir à un rapport ancien et intime à l'écriture littéraire du spirituel, celui-ci soit-il religieux, séculier ou post séculier, et à sa faible exploitation par nos auteurs. Auerbach (1942) a rappelé dans Mimèsis, et les historiens de l'art dans de nombreux essais, l'importance du dogme de l'incarnation dans la culture chrétienne: Dieu s'est fait homme, dans un corps qui a souffert et qui est mort ...d'où 
toute une culture de la représentation figurative, malgré diverses crises iconoclastes. Le monde musulman contemporain connaît et partage largement cette culture, sauf dans ses manifestations radicales. Le langage, jusque dans sa dimension la plus représentative, est donc susceptible de réactiver une dimension spirituelle, sans nécessairement passer par la dimension idéographique qui a longtemps caractérisé la littérature européenne, du moins sans passer par l'allégorie ou le surnaturel - mais cela implique de suggérer aussi un temps à venir - pas nécessairement celui du jugement dernier et du royaume de Dieu, mais celui de réalisations possibles. Dans L'Âge séculier, Charles Taylor montre combien la pensée chrétienne a su se marier aux philosophies antiques et aux mentalités des différentes sociétés, évoluant dans leur rapport à la nature et aux autres, au point de croiser des pensées politiques, ie d'inspirer mais aussi d'être influencée par elles. Il s'arrête ainsi sur la fin du Moyen-Âge, quand le respect des choses créées par Dieu devient conscience de devoir faire s'épanouir les formes potentielles créées par lui, avant d'évoquer, bien sûr la Réforme protestante, puis le déisme du XVIIIe siècle. Pierre Musso (2017), de son côté, a proposé une synthèse des rapports entre la vie monastique et le monde de l'entreprise, en insistant sur la règle de St Benoît parfois ramenée à la consigne "ora et labora ". Cette première étape de l'histoire occidentale invite à nuancer l'opposition entre les deux sectes chrétiennes : le protestantisme des pays du nord de l'Europe n'a pas l'apanage de l'action dans le monde, si l'on ose dire ${ }^{16}$. Et le langage peut représenter et figurer l'empirique, le séculier, et leur dépassement par l'image.

La littérature française semble peiner à envisager pareils futurs, c'est-à-dire aussi à envisager du commun, sur les plans sociaux et politiques - ce qui reflète le désarroi du XXIe siècle non pas tant après le désenchantement religieux que suite aux échecs des utopies de gauche et de la pensée socialisante du libéralisme originel. Pourquoi cependant la fiction ne s'inscrit-elle pas dans un post séculier constructif? Une réponse passerait peut-être par l'étude des fondements culturels du réalisme magique, qui s'est développé d'abord dans la moitié sud du continent américain, avant de gagner toute la littérature anglophone, et qui joue par excellence sur les dimensions plurielles des langages de la fiction. Peut-être faut-il mieux se placer entre le démon du textualisme et l'exigence d'un réalisme social trop empirique et trop rationnel, ce à quoi Marie N'Diaye s'essaie parfois, par exemple? La pensée française de la laïcité nous a-t-elle détournés du spirituel, au point que nous ne sachions plus traiter celui-ci, que nous n'osions plus l'adapter? Boualem Sansal en est un défenseur intelligent, qui met surtout en garde contre les dérives dogmatiques plus qu'il n'imagine du post séculier.

Paradoxalement, c'est peut-être du côté de l'essai historico-autobiographique, soit une forme résolument hybride, et non dans une fiction mariant souplement deux dimensions, que le lecteur français trouvera une réflexion sur le religieux lié au politique, au « commun » et à la vie singulière : dans Composition française. Retour sur une enfance bretonne, l'historienne Mona Ozouf (2009), narre et explique son enfance dans une Bretagne prise entre catholicisme et gauche internationaliste, pour orienter ses réflexions sur les diverses communautés vivant en France, sur le sens d'une identité « multiple », et sur les positions que cela invite à prendre dans la société (sur la "question du voile", par exemple). À l'Histoire (et au Droit) de devenir force(s) de proposition imaginante ${ }^{17}$ ? 
On en conclura que la littérature du XXIe siècle, mariant plus que jamais réalités mondaines socio-politiques et désir de spiritualité éthique constitue un terrain de choix pour l'imagination post séculière, et celle-ci se laisse deviner chez les auteurs de notre corpus. La fiction contemporaine française, en revanche, et malgré l'histoire des religions et de la pensée politique, n'ose pas les hypothèses poétiques d'un mouvement comme le réalisme magique, repris par la World Literature. Ce qui signifie simplement que la pensée du monde continue de défier les formes du roman de langue française, encore et toujours en mutation.

\section{BIBLIOGRAPHIE}

ANDERSON, Benedict (1996 [1983]). L'Imaginaire national. Réflexions sur l'origine et l'essor du nationalisme. Paris : La Découverte.

AUDARD, Catherine (2009). Qu'est-ce que le libéralisme? Éthique, politique, société. Paris : Gallimard, « Folio essais ».

AUERBACH, Éric (1942). Mimésis. Paris: Gallimard, « Tel ».

BEAUVOIR, Simone de et al. (1965). Que peut la littérature ? Présentation par Yves Buin. Paris: Union Générale d'Éditions, collection «10/18».

BELL, Thomas Richard (2015). The Postsecular traces of Transcendence in Contemporary German

Literature. Thèse de philosophie. Washington: University of Washington. [consulté le 28/8/2018]

URL https://digital.lib.washington.edu/researchworks/bitstream/handle/1773/33949/

Bell_washington_0250E_14594.pdf ?sequence =1

CAMILLERI, Andrea (2018). Noli me tangere. Traduit par Serge Quadruppani. Paris : Éditions Métailié.

CARRÈrE, Emmanuel (2010 [2009]). D’Autres Vies que la mienne. Paris : Folio Gallimard.

CARRÈRE, Emmanuel (2014). Le Royaume. Paris : P.O.L.

CASTINO, Didier (2015). Après le silence. Paris : Liana Levi.

CHAMOISEAU, Patrick (2012). L'Empreinte à Crusoë. Paris : Gallimard.

CHEAH, Pheng (2004). Spectral Nationality: Passages of Freedom from Kant to Postcolonial Literature of Liberation. Columbia : Columbia University Press.

DAOUD, Kamel (2016). Meursault, contre-enquête. Paris : Gallimard.

DELMAS-MARTY, Mireille (2011-2014). Forces imaginantes du Droit. 4 tomes. Paris : Seuil.

GAUCHET, Marcel (1985). Le Désenchantement du monde : une histoire politique de la religion. Paris :

Gallimard.

GEFEN, Alexandre (2017). Réparer le monde. La littérature française face au XXIe siècle. Paris : Corti. 
GINZBURG, Carlo (2013 [2008]). « Peur révérence terreur » (2008), in Martin Rueff (trad. de l'anglais et de l'italien). Peur révérence terreur. Quatre essais d'iconographie politique. Paris : Les Presses du réel.

HABERMAS, Jürgen (2008). « Qu'est-ce qu'une société « post-séculière »? », Le Débat, n 152, 5, pp. $4-15$.

HADOT, Pierre (2002). Exercices spirituels et philosophie antique. Paris : Albin Michel.

HADOT, Pierre (2008). N'oublie pas de vivre. Paris : Albin Michel.

HAENEL, Yannick (2007). Cercles. Paris : Gallimard.

HAENEL, Yannick (2013). Les Renards pâles. Paris : Gallimard.

LEFRANC, Alban (2006). Des Foules, des bouches, des armes. Paris : Melville/Léo Scheer.

HOUELLEBECQ, Michel (1998). Les Particules élémentaires. Paris : Flammarion.

HOUELLEBECQ, Michel (2015). Soumission. Paris : Flammarion.

JOSEPH-VILAIN, Mélanie, MISRAHI-BARAK, Judith (éd.), (2010). Postcolonial ghosts. Fantômes postcoloniaux - avec des poèmes de Gerry Turcotte. Montpellier : Presses Universitaires de la Méditerranée.

MAVELLI, Luca, PETITO, Fabio (2012). « The Post Secularism in International Relations: an

overview ", Review of International Studies, $\mathrm{n}^{\circ} 38$, p. 931-942.

Musso, Pierre (2017). La Religion industrielle : monastère, manufacture, usine. Une généalogie de l'entreprise. Paris : Fayard.

OzoUf, Mona (2009). Composition française. Retour sur une enfance bretonne. Paris : Gallimard.

PAVEL, Thomas (2003). La Pensée du roman. Paris : Gallimard.

PILAR BLANCO, Maria del, PEEREN, Esther (2013). The Spectralities Reader: Ghosts and Haunting in Contemporary Cultural Theory. London \& New York: Bloomsbury.

RATTI, Manav (2013). The Postsecular Imagination. Postcolonialism, Religion and Literature. Routledge Research in Postcolonial Literatures, vol. 45. New York \& Oxfordshire: Routledge.

RIBOULET, Mathieu (2015). Entre les deux il n'y a rien. Paris : Verdier.

ROLIN, Jean (1994). Joséphine. Paris : Gallimard.

ROLIN, Jean (1995). Zones. Paris : Gallimard.

RoLIN, Jean (2003). Chrétiens. Paris : P.O.L.

ROLIN, Jean (2015). Savannah. Paris : P.O.L.

RoLIN, Jean (2015). Les Événements. Paris : P.O.L.

SABOT, Philippe (2002). Pour une « hantologie » littéraire, Philosophie et littérature. Approches et enjeux d'une question. Paris : PUF, « philosophies ».

SANSAL, Boualem (2015). 2084, la fin du monde. Paris : Gallimard.

SARRAUTE, Nathalie (1956). L'Ère du soupçon. Paris : Gallimard.

TAYLOR, Charles (2007). L'Âge séculier. Paris : Seuil, « Les Livres du nouveau monde », trad. P. Savidan, 2011. 
TRAVERSO, Enzo (2016). Mélancolie de gauche : la force d'une tradition cachée (XIXe-XXIe siècles). Paris : La Découverte.

VASSET, Philippe (2013). La Conjuration. Paris : Fayard.

VASSET, Philippe (2016). La Légende. Paris : Fayard.

WEBER, Max (1964 [1920]). Éthique protestant et esprit du capitalisme. Paris : Plon.

WEBER, Max (1971 [1921]). Économie et société. Paris : Plon.

ZENETTI, Marie-Jeanne (2013). « Les factographies : déplacements des discours de l'histoire », Fabula, colloques en ligne, Littérature et histoire en débats [page consultée le 28/8/2018] URL : http:// www.fabula.org/colloques/document2123.php

\section{NOTES}

1. Emmanuel Macron, président de la France, a eu une entrevue avec lui le 26 juin 2018.

2. Voir, pour exemple, le soutien officiel de Vladimir Poutine à la construction de la monumentale cathédrale orthodoxe du Quai Branly.

3. Pour la critique du désenchantement dont Marcel Gauchet (1985) fait un historique, voir Carlo Ginzburg (2013).

4. Voir Zenetti (2013).

5. Alexandre Gefen (2017) décèle même dans la littérature française «face au XXIe siècle " l'ambition de Réparer le monde.

6. Voir Hadot $(2002,2008)$.

7. En renouvelant le débat lancé dans Beauvoir (1965).

8. Voir, par exemple, Pilar Blanco, Peeren (2013), Cheah (2004), Sabot (2002) et Joseph-Vilain, Misrahi-Barak (2010).

9. https://www.franceculture.fr/emissions/lecriture-est-un-sport-comme-les-autres/emmanuelcarrere-et-le-yoga.

10. «Vierge sainte, au milieu de vos jours glorieux, n'oubliez pas les tristesses de la terre (...) Ayez pitié de ceux qui s'aimaient et qui ont été séparés. Ayez pitié de la faiblesse de notre foi... » (Rolin, 1994 : 87).

11. Excipit de La Conjuration (Vasset, 2013 : 206).

12. Sur le plan de la politique et de l'histoire des idées, Enzo Traverso (2016) en a rendu compte.

13. Je me permets de renvoyer à Catherine Grall: http://www.raison-publique.fr/ auteur2185.html.

14. Le titre cite Ursula Meinhoff, membre actif de la Fraction Armée Rouge.

15. Il s'agit de Salman Rushdie, Michael Ondaatje, Amitav Gosh, Shauna Sing Baldwin, Allan Sealy.

16. Et la religion juive dispose que les hommes, après leur séparation d'avec Dieu, doivent rechercher son alliance et « réparer le monde. "

17. Cf les quatre tomes consacrés par Mireille Delmas-Marty (2011-2014) aux Forces imaginantes du Droit. 


\section{RÉSUMÉS}

Plusieurs romans français contemporains mentionnent les religions chrétienne et musulmane, entremêlant réalisme et désir de transcendance. Les politiques libérales, oublieuses de leur origine philosophique qui reconnaissait la valeur de tout individu au sein de la société, conduisent à un mauvais traitement de celui-ci dans les intrigues. Les romanciers de notre corpus, contrairement à une littérature dite "world», connaissent des difficultés pour imaginer un post séculier constructif dans leur fiction.

A few contemporary French novels mention christian and muslim religion, mixing a new realism (developed since the 1980's in Western literature) with the need for transcendance. The liberal politics, forgeting their origins, that included the value of individualism in the society, leave the characters unsatisfied, but the novelists of our corpus, unlike some world literature, experience difficulties to imagine a constructive post secularism in their fictions.

\section{INDEX}

Mots-clés : littérature française, littérature générale, post séculier, religion, réalisme, contemporain

Keywords : french literature, comparative literature, post secularism, religion, contemporary realism

\section{AUTEUR}

\section{CATHERINE GRALL}

Université de Picardie Jules Verne grallthecat[at]gmail.com 